\title{
Finite-time Consensus for Multi-agent Systems via Nonlinear Control Protocols
}

\author{
Ya-Kun Zhu ${ }^{1} \quad$ Xin-Ping Guan ${ }^{1,2} \quad$ Xiao-Yuan Luo ${ }^{1}$ \\ ${ }^{1}$ Institute of Electrical Engineering, Yanshan University, Qinhuangdao 066004, China \\ ${ }^{2}$ School of Electronic, Information and Electrical Engineering, Shanghai Jiao Tong University, Shanghai 200240, China
}

\begin{abstract}
This paper investigates the finite-time consensus problem of multi-agent systems with single and double integrator dynamics, respectively. Some novel nonlinear protocols are constructed for first-order and second-order leader-follower multi-agent systems, respectively. Based on the finite-time control technique, the graph theory and Lyapunov direct method, some theoretical results are proposed to ensure that the states of all the follower agents can converge to its leader agent's state in finite time. Finally, some simulation results are presented to illustrate the effectiveness of our theoretical results.
\end{abstract}

Keywords: Finite-time consensus, multi-agent systems, nonlinear control protocol, leader-follower, single and double integrator dynamics.

\section{Introduction}

In recent years, the study of consensus problems for multi-agent systems has received a great deal of attentions and considerable research efforts in various fields, partially due to the broad applications in motion control of multiple mobile robots ${ }^{[1]}$, formation control ${ }^{[2]}$, and target tracking and obstacle avoidance ${ }^{[3]}$, etc. Consensus means that a group of agents reaches an agreement on a common value by communicating with their neighbors.

Consensus problems have been studied from many different aspects. Ren et al. ${ }^{[4,5]}$ studied the consensus problems of continuous systems and discrete-time systems, respectively. Average consensus, power-mean consensus and $\max / \mathrm{min}$ consensus were investigated respectively ${ }^{[6,7]}$. Bouso et al. ${ }^{[6]}$ considered stationary consensus protocols for networks of dynamic agents with fixed topologies. Cortes ${ }^{[7]}$ systematically derived necessary and sufficient conditions on a distributed algorithm that asymptotically achieves consensus states which are general continuous functions of the initial states of all agents. It means that if we change the initial states of the agents, the consensus state changes. Systems with switching network topologies and communication delays were studied in [8]. However, the common value, which is called consensus state, usually depends on the initial state. And in most of the existing works, the consensus state is usually assumed to be a constant, a weighted average of the initial conditions or a function of initial conditions. In leader-follower multi-agent system, the followers are all required to reach an agreement on the leader's states, and the leader is usually independent of their followers, but influences the followers. Hence, the control objective can

Manuscript received June 28, 2012; revised March 11, 2013

This work was supported by National Basic Research Program of China (973 Program) (No. 2010CB731800), National Natural Science Foundation of China (Nos. 60934003 and 61074065), Natural Science Foundation of Hebei Province (No. F2012203119)). be realized by controlling the leader only. And the control objective can reach the desired value besides a weighted average or a function of initial conditions.

Another important study of the consensus problem is the convergence rate. $\mathrm{Hu}^{[9]}$ investigated the problem of robust consensus tracking for a class of second-order multiagent dynamic systems with disturbances and unmodeled agent dynamics. By designing continuous distributed consensus protocols, he has proved that all the agents can globally asymptotically reach consensus with the desired states. However, most of the existing consensus control algorithms for multi-agent systems are asymptotic consensus algorithms, which means that the convergence rate is at best exponential with infinite settling time ${ }^{[10]}$. Then, finite-time consensus algorithms are desirable. Besides a faster convergence rate, the systems under finite-time control usually demonstrate better disturbance rejection properties. Due to these superiorities, several kinds of finite-time consensus protocols have been developed ${ }^{[11-13]}$.

Inspired by these facts, it is significant and necessary to study the finite-time consensus problem of the leaderfollower multi-agent systems via nonlinear control protocols. In this paper, we study the finite-time consensus problems of first-order and second-order leader-follower multiagent systems respectively. Compared with these mentioned literatures, the contributions of this paper mainly lie in three aspects. Firstly, novel nonlinear control protocols are designed for first-order and second-order multi-agent systems, respectively. Secondly, the agents can converge to its leader agent's state no matter the initial states change or not. Thirdly, the consensus can be achieved in finite time.

The rest of this paper is organized as follows. Section 2 introduces preliminaries and some lemmas. Section 3 discusses the nonlinear control protocols for the multi-agent systems with single and double integrator dynamics, respectively. Sufficient finite-time consensus criteria are obtained 
for multi-agent systems with single and double integrator dynamics. Some numerical examples are given in Section 4. Finally, we present the conclusions in Section 5 .

Notations. Throughout this paper, $\mathbf{R}$ and $\mathbf{R}^{+}$stand for the set of real numbers and positive real numbers, respectively. $\mathbf{R}^{n}$ denotes the $n$-dimensional real vector space. $\mathbf{R}^{n \times n}$ is the set of $n \times n$ matrices. $1_{n}$ (or $0_{n}$ ) is a vector with all the elements being one (or zero). The superscript $\mathrm{T}$ stands for the transpose of a matrix.

\section{Preliminaries and some lemmas}

\subsection{Graph theory}

For multi-agent systems, we assume that each agent is a node and the information exchange between agents can be naturally modeled by the undirected weighted graph $G=\{V, E, A\}$, where the node indexes belong to a finite index set $\Gamma=\{1,2, \cdots, n\}, V=\left\{v_{i}\right\}$ is the set of agents, $E \subseteq V \times V$ is the set of links between the agents, and $A$ is the corresponding weighted adjacency matrix. The adjacency matrix $A=\left[a_{i j}\right] \in \mathbf{R}^{n \times n}$ is defined such that $a_{i j}>0$ if $\left(v_{j}, v_{i}\right) \in E$, while $a_{i j}=0$ if $\left(v_{j}, v_{i}\right) \notin E$, and $a_{i i}=0$ for all $i \in \Gamma$. The set of neighbors of agent $v_{i}$ is denoted by $N_{i}=\left\{v_{j}:\left(v_{j}, v_{i}\right) \in E\right\}$. The degree of agent $v_{i}$ is defined as $\operatorname{deg}\left(v_{i}\right)=d_{i}=\sum_{j=1}^{n} a_{i j}=\sum_{j \in N_{i}} a_{i j}$. Then, the degree matrix of graph $G$ is $D=\operatorname{diag}\left\{d_{1}, \cdots, d_{n}\right\}$ and the Laplacian matrix is $L=D-A$.

\subsection{Some lemmas and Lyapunov theory for finite-time stability}

Lemma $\mathbf{1}^{[14]}$. Suppose function $\phi: \mathbf{R}^{2} \rightarrow \mathbf{R}$ satisfies $\phi\left(x_{i}, x_{j}\right)=-\phi\left(x_{j}, x_{i}\right), i, j \in \Gamma, i \neq j$. Then, for any undirected graph $G$ and a set of numbers $y_{1}, y_{2}, \cdots, y_{N}$,

$$
\sum_{i=1}^{N} \sum_{j \in N_{i}} a_{i j} y_{i} \phi\left(x_{j}, x_{i}\right)=-\frac{1}{2} \sum_{\left(v_{i}, v_{j}\right) \in E} a_{i j}\left(y_{j}-y_{i}\right) \phi\left(x_{j}, x_{i}\right) .
$$

Lemma $\mathbf{2}^{[15]}$. For $x_{i} \in \mathbf{R}, i=1, \cdots, n, 0<p \leqslant 1$, then

$$
\left(\sum_{i=1}^{n}\left|x_{i}\right|\right)^{p} \leqslant \sum_{i=1}^{n}\left|x_{i}\right|^{p} \leqslant n^{1-p}\left(\sum_{i=1}^{n}\left|x_{i}\right|\right)^{p}
$$

Lemma $3^{[16]}$. For a connected undirected graph $G$, the Laplacian matrix $L$ of $G$ has the following properties. $\quad x^{\mathrm{T}} L x=\frac{1}{2} \sum_{i, j=1}^{n} a_{i j}\left(x_{j}-x_{i}\right)^{2}=$ $\frac{1}{2} \sum_{i=1}^{N} \sum_{j \in N_{i}} a_{i j}\left(x_{j}-x_{i}\right)^{2}$ for any $x=\left[x_{1}, \cdots, x_{n}\right]^{\mathrm{T}} \in$ $\mathbf{R}^{n}$, which implies that $L$ is positive semi-definite. 0 is a simple eigenvalue of $L$ and $1_{n}$ is the associated eigenvector. Assume that the eigenvalues of $L$ are denoted by $0, \lambda_{2}, \cdots, \lambda_{n}$ satisfying $0 \leqslant \lambda_{2} \leqslant \cdots \leqslant \lambda_{n}$. Then, the second smallest eigenvalue $\lambda_{2}>0$. Furthermore, if $1_{n}^{\mathrm{T}} x=0$, then $x^{\mathrm{T}} L x \geqslant \lambda_{2} x^{\mathrm{T}} x$.

Lemma 4. Suppose function $\phi: \mathbf{R}^{2} \rightarrow \mathbf{R}^{+}$satisfies $\phi\left(x_{i}, x_{j}\right)=\phi\left(x_{j}, x_{i}\right), i, j \in \Gamma, i \neq j$. Then for any undi- rected graph $G$ and a set of numbers $y_{1}, y_{2}, \cdots, y_{N}$,

$$
\sum_{i=1}^{N} \sum_{j \in N_{i}} a_{i j}\left|y_{i}\right| \phi\left(x_{j}, x_{i}\right) \geqslant \frac{1}{2} \sum_{i=1}^{N} \sum_{j \in N_{i}} a_{i j}\left|y_{j}-y_{i}\right| \phi\left(x_{j}, x_{i}\right) .
$$

Proof. From the definition of an undirected graph and the assumption of function $\phi$, we can obtain that

$$
\begin{aligned}
& \sum_{i=1}^{N} \sum_{j \in N_{i}} a_{i j}\left|y_{i}\right| \phi\left(x_{j}, x_{i}\right)= \\
& \frac{1}{2}\left[\sum_{\left(v_{i}, v_{j}\right) \in E} a_{i j}\left|y_{i}\right| \phi\left(x_{j}, x_{i}\right)+\sum_{\left(v_{i}, v_{j}\right) \in E} a_{i j}\left|y_{i}\right| \phi\left(x_{j}, x_{i}\right)\right]= \\
& \frac{1}{2}\left[\sum_{\left(v_{i}, v_{j}\right) \in E} a_{i j}\left|y_{i}\right| \phi\left(x_{j}, x_{i}\right)+\sum_{\left(v_{i}, v_{j}\right) \in E} a_{j i}\left|y_{j}\right| \phi\left(x_{i}, x_{j}\right)\right]= \\
& \frac{1}{2}\left[\sum_{\left(v_{i}, v_{j}\right) \in E} a_{i j}\left|y_{i}\right| \phi\left(x_{j}, x_{i}\right)+\sum_{\left(v_{i}, v_{j}\right) \in E} a_{i j}\left|y_{j}\right| \phi\left(x_{j}, x_{i}\right)\right]= \\
& \frac{1}{2} \sum_{\left(v_{i}, v_{j}\right) \in E} a_{i j}\left(\left|y_{i}\right|+\left|y_{j}\right|\right) \phi\left(x_{j}, x_{i}\right) \geqslant \\
& \frac{1}{2} \sum_{i=1}^{N} \sum_{j \in N_{i}} a_{i j}\left|y_{j}-y_{i}\right| \phi\left(x_{j}, x_{i}\right) .
\end{aligned}
$$

It is well known that a sufficient condition for the existence of a unique solution of a nonlinear differential equation is that the function is locally Lipschitz continuous. The solution of such nonlinear differential equation can have at most asymptotic convergence rate. Since finite-time stability guarantees that every system state reaches the system origin in a finite time, the finite-time stability has a much stronger requirement than the asymptotic stability. The following lemma presents sufficient conditions for the finite-time stability.

Lemma $\mathbf{5}^{[17]}$. Considering the non-Lipschitz continuous nonlinear system $\dot{x}=f(x), f(0)=0, x \in \mathbf{R}^{n}$, there exist a positive definite continuous function $V(x): U \rightarrow \mathbf{R}$, real numbers $c>0$ and $\alpha \in(0,1)$, and an open neighborhood $U_{0} \subset U$ of the origin such that $\dot{V}(x)+c(V(x))^{\alpha} \leqslant 0$, $x \in U_{0} \backslash\{0\}$. Then, $V(x)$ approaches 0 in finite time. In addition, the finite settling time $T$ satisfies that $T \leqslant$ $\frac{(V(x(0)))^{1-\alpha}}{c(1-\alpha)}$.

\section{Main results}

For simplicity of presentation, we assume that the states of all agents are in a one dimensional space. However, the results of this paper are still valid for multiple high dimensional agents by the introduction of the Kronecker product and appropriately rewriting the protocol.

\subsection{Finite-time consensus for single inte- grator dynamics}

Consider a distributed system consisting of $n$ agents. Each agent has the single integrator dynamics given by

$$
\dot{x}_{i}(t)=u_{i}(t), \quad i \in \Gamma, \quad \Gamma=\{1,2, \cdots, n\}
$$


where $x_{i}(t) \in \mathbf{R}$ is the state of the $i$-th agent, and $u_{i}(t) \in \mathbf{R}$ is the feedback control law to be designed, which depends only on the states of agent $i$ and its neighbors.

The dynamics of the leader indexed by 0 is described as

$$
\dot{x}_{0}(t)=u_{0}(t), \quad x_{0}(t) \in \mathbf{R} .
$$

We design a class of nonlinear consensus protocol which is described by

$$
\begin{aligned}
u_{i}(t)= & \sum_{j \in N_{i}} a_{i j} \psi\left(x_{j}-x_{i}\right)\left|x_{j}(t)-x_{i}(t)\right|^{\alpha-1}+ \\
& \dot{x}_{0}(t)-b_{i}\left(x_{i}(t)-x_{0}(t)\right)
\end{aligned}
$$

where $b_{i} \geqslant 0$ and the function $\psi(\cdot)$ satisfies Assumption 1 , which holds throughout this paper.

Assumption 1. Function $\psi(y)$ satisfies that $y \psi(y)>0$, $\forall y \in R \backslash\{0\}, \psi(0)=0$. And $\psi(y) \geqslant r y^{q}, \forall y \in \mathbf{R}$, where $r>0, q=\frac{q_{1}}{q_{2}}<1, q_{1}$ and $q_{2}$ are positive odd integers.

Theorem 1. Suppose the graph $G$ is undirected and connected, and $\max \{1,-q\}<\alpha<2-q$. Then, the multiagent system (1) can solve the consensus problem in finite time with the nonlinear consensus protocol (2).

Proof. Substituting the nonlinear consensus protocol (2) into the multi-agent system (1) yields

$$
\begin{aligned}
\dot{x}_{i}(t)= & \sum_{j \in N_{i}} a_{i j} \psi\left(x_{j}-x_{i}\right)\left|x_{j}(t)-x_{i}(t)\right|^{\alpha-1}+ \\
& \dot{x}_{0}(t)-b_{i}\left(x_{i}(t)-x_{0}(t)\right) .
\end{aligned}
$$

Let $e_{i}(t)=x_{i}(t)-x_{0}(t)$, then the error systems can be obtained from system (3), which is described as

$$
\dot{e}_{i}(t)=\sum_{j \in N_{i}} a_{i j} \psi\left(e_{j}-e_{i}\right)\left|e_{j}(t)-e_{i}(t)\right|^{\alpha-1}-b_{i} e_{i}(t)
$$

Consider the Lyapunov function

$$
V(e(t))=\frac{1}{2} \sum_{i \in \Gamma} e_{i}^{2}(t)
$$

Obviously, $V(e(t))$ is a positive definite function with respect to $e(t)$. Differentiating the Lyapunov function with respect to $t$ and according to Lemma 1, we have

$$
\begin{aligned}
& \dot{V}(e(t))=\sum_{i \in \Gamma} e_{i}(t) \dot{e}_{i}(t)= \\
& \sum_{i \in \Gamma} e_{i}(t)\left(\sum_{j \in N_{i}} a_{i j} \psi\left(e_{j}-e_{i}\right)\left|e_{j}(t)-e_{i}(t)\right|^{\alpha-1}-b_{i} e_{i}(t)\right)= \\
& -\frac{1}{2} \sum_{i \in \Gamma} \sum_{j \in N_{i}}\left(e_{j}(t)-e_{i}(t)\right) a_{i j} \psi\left(e_{j}(t)-e_{i}(t)\right) \times \\
& \left|e_{j}(t)-e_{i}(t)\right|^{\alpha-1}-\sum_{i \in \Gamma} b_{i} e_{i}^{2}(t) .
\end{aligned}
$$

We can see that $\dot{V}(e(t)) \leqslant 0$ due to Assumption 1. More- over, we can also obtain that

$$
\begin{aligned}
& \dot{V}(e(t)) \leqslant \\
& \quad-\frac{r}{2} \sum_{i \in \Gamma} \sum_{j \in N_{i}} a_{i j}\left(e_{j}(t)-e_{i}(t)\right)\left(e_{j}(t)-e_{i}(t)\right)^{q} \times \\
& \left|e_{j}(t)-e_{i}(t)\right|^{\alpha-1}= \\
& \quad-\frac{r}{2} \sum_{i \in \Gamma} \sum_{j \in N_{i}} a_{i j}\left|e_{j}(t)-e_{i}(t)\right|^{\alpha+q}= \\
& \quad-\frac{r}{2} \sum_{i \in \Gamma} \sum_{j \in N_{i}} a_{i j}\left(\left(e_{j}(t)-e_{i}(t)\right)^{2}\right)^{\frac{\alpha+q}{2}}= \\
& \quad-\frac{r}{2} \sum_{i \in \Gamma} \sum_{j \in N_{i}}\left(a_{i j}^{\frac{2}{\alpha+q}}\left(e_{j}(t)-e_{i}(t)\right)^{2}\right)^{\frac{\alpha+q}{2}} .
\end{aligned}
$$

From Lemmas 2 and 3, we have

$$
\begin{aligned}
& \dot{V}(e(t)) \leqslant \\
& -\frac{r}{2}\left(\sum_{i \in \Gamma} \sum_{j \in N_{i}} a_{i j}^{\frac{2}{\alpha+q}}\left(e_{j}(t)-e_{i}(t)\right)^{2}\right)^{\frac{\alpha+q}{2}}= \\
& \quad-\frac{r}{2}\left(2 e^{\mathrm{T}}(t) L_{B} e(t)\right)^{\frac{\alpha+q}{2}} \leqslant \\
& \quad-\frac{r}{2}\left(4 \lambda_{B} V(t)\right)^{\frac{\alpha+q}{2}}= \\
& \quad-2^{\alpha-1+q} r \lambda_{B}^{\frac{\alpha+q}{2}} V^{\frac{\alpha+q}{2}}(t)
\end{aligned}
$$

where $\lambda_{B}=\lambda_{2}\left(L_{B}\right), L_{B}$ is the Laplacian matrix of graph $G(B)$, and $B=\left[a_{i j}^{\frac{2}{\alpha+q}}\right] \in \mathbf{R}^{n \times n}$.

So, we have

$$
\dot{V}(e(t))+2^{\alpha-1+q} r \lambda_{B}^{\frac{\alpha+q}{2}} \cdot V^{\frac{\alpha+q}{2}}(e(t)) \leqslant 0 .
$$

Consequently, consensus can be achieved in finite time according to Lemma 5. Moreover, we can obtain that $T \leqslant \frac{V(e(0))^{1-\frac{\alpha+q}{2}}}{2^{\alpha-1+q_{r} \lambda_{B}^{\frac{\alpha+q}{2}}\left(1-\frac{\alpha+q}{2}\right)} .}$

\subsection{Finite-time consensus for double inte- grator dynamics}

Consider the linear double integrator dynamical multiagent systems

$$
\left\{\begin{array}{c}
\dot{x}_{i}=v_{i} \\
\dot{v}_{i}=u_{i}
\end{array}, i \in \Gamma\right.
$$

where $x_{i} \in \mathbf{R}$ and $v_{i} \in \mathbf{R}$ are the position and velocity of the $i$-th agent, $u_{i}$ is the feedback control protocol to be designed.

The dynamics of the leader indexed by 0 is described as

$$
\begin{cases}\dot{x}_{0}=v_{0}, & x_{0} \in \mathbf{R} \\ \dot{v}_{0}=u_{0}, & v_{0} \in \mathbf{R} .\end{cases}
$$

Now, we present a class of nonlinear consensus protocol 
which is described as

$$
\begin{aligned}
u_{i}= & -\left(\beta_{1} \sum_{j \in N_{i}} a_{i j} \psi\left(\left|x_{j}-x_{i}\right|\right)\left|x_{j}-x_{i}\right|^{\alpha-1}+\right. \\
& \beta_{2} \sum_{j \in N_{i}} a_{i j} \psi\left(\left|v_{j}-v_{i}\right|\right)\left|v_{j}-v_{i}\right|^{\alpha-1}+ \\
& \left.c_{i}\left|v_{i}-v_{0}\right|\right) \operatorname{sgn}\left(x_{i}-x_{0}+v_{i}-v_{0}\right)+ \\
& \dot{v}_{0}-\left(v_{i}-v_{0}\right)
\end{aligned}
$$

where $A=\left[a_{i j}\right]_{n \times n}$ is the weighted adjacency matrix, $c_{i} \geqslant 0, \beta_{1}$ and $\beta_{2}$ are positive constants denoting the feedback gains, $\beta=\min \left\{\beta_{1}, \beta_{2}\right\}$, $\operatorname{sgn}(\cdot)$ is the sign function, the interaction function $\psi(\cdot)$ satisfies the Assumption 1 in Section 3.1.

Theorem 2. Suppose the communication network $G$ is undirected and connected, and $\max \{1,-q\}<\alpha<2-q$. Then, the second-order dynamical multi-agent system (7) can solve the consensus problem with the nonlinear consensus protocol (8) in finite time.

Proof. Let $\bar{x}_{i}(t)=x_{i}(t)-x_{0}(t), \bar{v}_{i}(t)=v_{i}(t)-v_{0}(t)$. Substituting the nonlinear consensus protocol (7) into the dynamic equations (8) yields

$$
\begin{aligned}
\dot{\bar{x}}_{i}= & \bar{v}_{i} \\
\dot{\bar{v}}_{i}= & -\left(\beta_{1} \sum_{j \in N_{i}} a_{i j} \psi\left(\left|\bar{x}_{j}-\bar{x}_{i}\right|\right)\left|\bar{x}_{j}-\bar{x}_{i}\right|^{\alpha-1}+\right. \\
& \beta_{2} \sum_{j \in N_{i}} a_{i j} \psi\left(\left|\bar{v}_{j}-\bar{v}_{i}\right|\right)\left|\bar{v}_{j}-\bar{v}_{i}\right|^{\alpha-1}+ \\
& \left.c_{i}\left|\bar{v}_{i}\right|\right) \operatorname{sgn}\left(\bar{x}_{i}+\bar{v}_{i}\right)-\bar{v}_{i} .
\end{aligned}
$$

Denote $s_{i}=\bar{x}_{i}+\bar{v}_{i}, S=\left(\begin{array}{llll}s_{1} & s_{2} & \cdots & s_{n}\end{array}\right)^{\mathrm{T}}$. Then we consider the following Lyapunov function

$$
V=\frac{1}{2} S^{\mathrm{T}} S=\frac{1}{2} \sum_{i \in \Gamma} s_{i}^{2}=\frac{1}{2} \sum_{i \in \Gamma}\left(\bar{x}_{i}+\bar{v}_{i}\right)^{2} .
$$

Differentiating the Lyapunov function with respect to $t$, we have

$$
\begin{aligned}
\dot{V}= & \sum_{i \in \Gamma} s_{i}\left(\dot{\bar{x}}_{i}+\dot{\bar{v}}_{i}\right)= \\
& \sum_{i \in \Gamma} s_{i}\left(\bar{v}_{i}-\left(\beta_{1} \sum_{j \in N_{i}} a_{i j} \psi\left(\left|\bar{x}_{j}-\bar{x}_{i}\right|\right)\left|\bar{x}_{j}-\bar{x}_{i}\right|^{\alpha-1}+\right.\right. \\
& \beta_{2} \sum_{j \in N_{i}} a_{i j} \psi\left(\left|\bar{v}_{j}-\bar{v}_{i}\right|\right)\left|\bar{v}_{j}-\bar{v}_{i}\right|^{\alpha-1}+ \\
& \left.\left.c_{i}\left|\bar{v}_{i}\right|\right) \operatorname{sgn}\left(s_{i}\right)-\bar{v}_{i}\right) \leqslant \\
& -\sum_{i \in \Gamma}\left|s_{i}\right|\left(\beta_{1} r \sum_{j \in N_{i}} a_{i j}\left|\bar{x}_{j}-\bar{x}_{i}\right|^{\alpha-1+q}+\right. \\
& \left.\beta_{2} r \sum_{j \in N_{i}} a_{i j}\left|\bar{v}_{j}-\bar{v}_{i}\right|^{\alpha-1+q}+c_{i}\left|\bar{v}_{i}\right|\right) \leqslant \\
& -\underline{\beta r} \sum_{i \in \Gamma}\left|s_{i}\right| \times
\end{aligned}
$$

$$
\left(\sum_{j \in N_{i}} a_{i j}\left(\left|\bar{x}_{j}-\bar{x}_{i}\right|^{\alpha-1+q}+\left|\bar{v}_{j}-\bar{v}_{i}\right|^{\alpha-1+q}\right)\right) \leqslant 0 .
$$

Note that $\max \{1,-q\}<\alpha<2-q$ yields $0<\alpha-1+q<1$. Using Lemmas 2 and 4 , we obtain that

$$
\begin{aligned}
\dot{V} \leqslant & -\underline{\beta} r \sum_{i \in \Gamma}\left|s_{i}\right|\left(\sum_{j \in N_{i}} a_{i j}\left(\left|\bar{x}_{j}-\bar{x}_{i}\right|+\left|\bar{v}_{j}-\bar{v}_{i}\right|\right)^{\alpha-1+q}\right) \leqslant \\
& -\underline{\beta} r \sum_{i \in \Gamma}\left|s_{i}\right|\left(\sum_{j \in N_{i}} a_{i j}\left|\bar{x}_{j}-\bar{x}_{i}+\bar{v}_{j}-\bar{v}_{i}\right|^{\alpha-1+q}\right)= \\
& -\underline{\beta r} \sum_{i \in \Gamma}\left|s_{i}\right|\left(\sum_{j \in N_{i}} a_{i j}\left|s_{j}-s_{i}\right|^{\alpha-1+q}\right) \leqslant \\
& -\frac{1}{2} \underline{\beta} \sum_{i \in \Gamma} \sum_{j \in N_{i}} a_{i j}\left|s_{j}-s_{i}\right|^{\alpha+q}= \\
& -\frac{1}{2} \underline{\beta} \sum_{i \in \Gamma} \sum_{j \in N_{i}}\left(a_{i j}^{\frac{2}{\alpha+q}}\left(s_{j}-s_{i}\right)^{2}\right)^{\frac{\alpha+q}{2}} \leqslant \\
& -\frac{1}{2} \underline{\beta} r\left(\sum_{i \in \Gamma} \sum_{j \in N_{i}} a_{i j}^{\frac{2}{\alpha+q}}\left(s_{j}-s_{i}\right)^{2}\right)^{\frac{\alpha+q}{2}} .
\end{aligned}
$$

Let $C=\left[a_{i j}^{\frac{2}{\alpha+q}}\right]_{n \times n} \in \mathbf{R}^{n \times n}, L_{C}$ be the Laplacian matrix of graph $G(C), \lambda_{2}\left(L_{C}\right)$ be the second smallest eigenvalue of $L_{C}$, and $\lambda_{C}=\lambda_{2}\left(L_{C}\right)$. From Lemma 3, we have

$$
\begin{aligned}
\dot{V} \leqslant & -\frac{1}{2} \underline{\beta}\left(2 S^{\mathrm{T}} L_{C} S\right)^{\frac{\alpha+q}{2}} \leqslant \\
& -\frac{1}{2} \underline{\beta}\left(4 \lambda_{2}\left(L_{C}\right) \cdot V\right)^{\frac{\alpha+q}{2}} \leqslant \\
& -2^{\alpha-1+q} \underline{\beta} r \lambda_{C}^{\frac{\alpha+q}{2}} V^{\frac{\alpha+q}{2}} .
\end{aligned}
$$

Let $c=2^{\alpha-1+q} \underline{\beta} r \lambda^{\frac{\alpha+q}{2}}>0$. Then, we can see that $\dot{V}+c V^{\frac{\alpha+q}{2}} \leqslant 0$. By Lemma 5 , one can obtain that finitetime consensus can be achieved with the settling time $T$ satisfying that $T \leqslant \frac{V(0)^{1-\frac{\alpha+q}{2}}}{c\left(1-\frac{\alpha+q}{2}\right)}$.

Remark 1. The nonlinear consensus protocol proposed here is different from the existing results (such as $[6,11-$ $14]$ ), and more general than the linear protocols (such as [16]). In particular, the consensus of all the agents can be achieved in finite time, which has more superiority than the asymptotic protocols (such as $[6,7]$ ).

\section{Simulation examples}

In this section, some simulation examples of nonlinear finite-time consensus are given to illustrate the effectiveness of the theoretical results in this paper.

\subsection{Simulation results for single integrator dynamics}

We consider a group of one leader agent and five follower agents with graph $G$ in Fig. 1. 


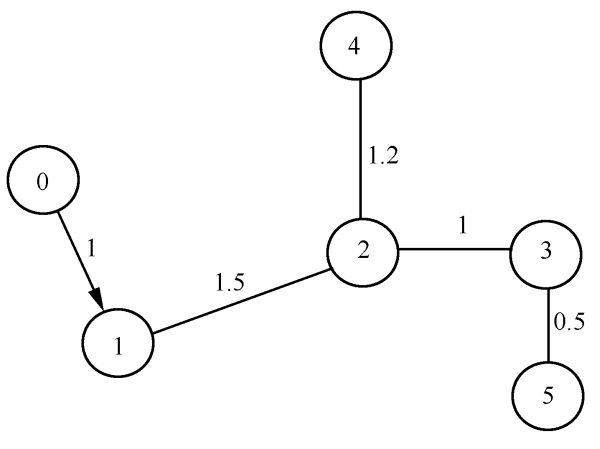

Fig. 1 The communication topology

We assume that $\psi(y)=e^{y} \times \operatorname{sgn}(y), \alpha=1.5$. It is clear that this situation cannot be modeled by any previously proposed linear consensus protocols. Three simulations are performed with three different consensus states.

Firstly, suppose that the first-order leader agent's state is $x_{0}(t)=2$. Fig. 2 shows that the follower agents' states can still converge to the leader agent's state in finite time under two different initial states $x(0)=[1,3.2,1.8,0.5,-1.2]^{\mathrm{T}}$ and $x(0)=[-1,0.2,1,2.5,0]^{\mathrm{T}}$.

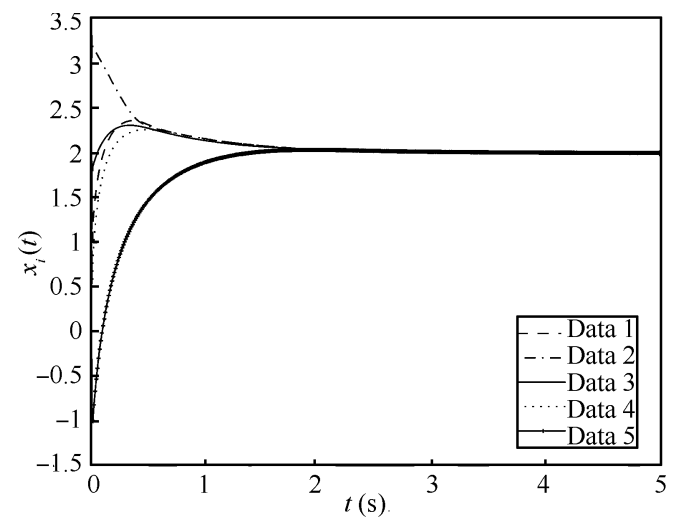

(a) State trajectories with $x(0)=[1,3.2,1.8,0.5,-1.2]^{\mathrm{T}}$

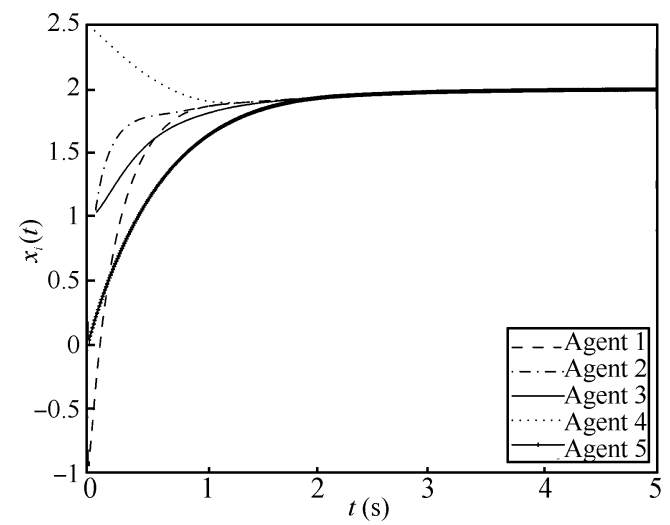

(b) State trajectories with $x(0)=[-1,0.2,1,2.5,0]^{\mathrm{T}}$

Fig. 2 The state trajectories of five agents with $x_{0}=2$

Then, we assume that firstly $x_{0}(t)=\sin (t)$ and then $x_{0}(t)=\cos (t)$ respectively. Figs. 3 and 4 show that the nonlinear consensus can be achieved in finite time. And the consensus state remains the same if we change the initial states, which means a better robustness compared with other literature whose consensus states closely relate to the agents' initial states. Figs. $2-4$ also indicate that our theoretical results are effective and have much faster convergence speed than the asymptotic results in $[6,7]$, etc.

\subsection{Simulation results for double integra- tor dynamics}

We consider a group of one leader agent and four follower agents with the undirected communication topology given in Fig. 5. $\psi(\cdot)$ and $\alpha$ are assumed to be the same as in Section 4.1 .

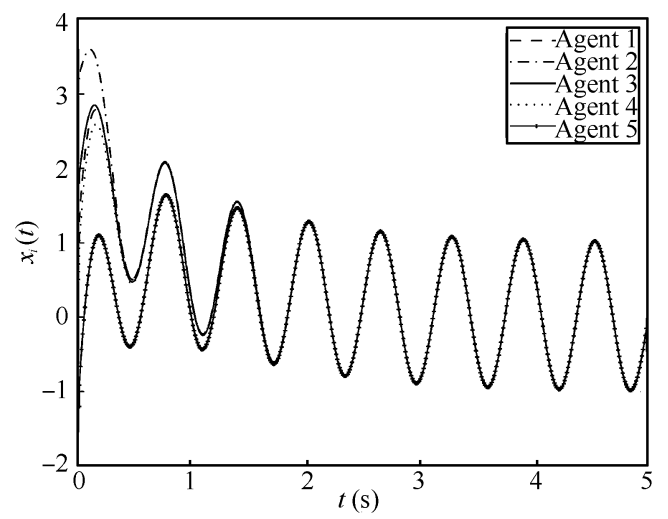

(a) State trajectories with $x(0)=[1,3.2,1.8,0.5,-1.2]^{\mathrm{T}}$

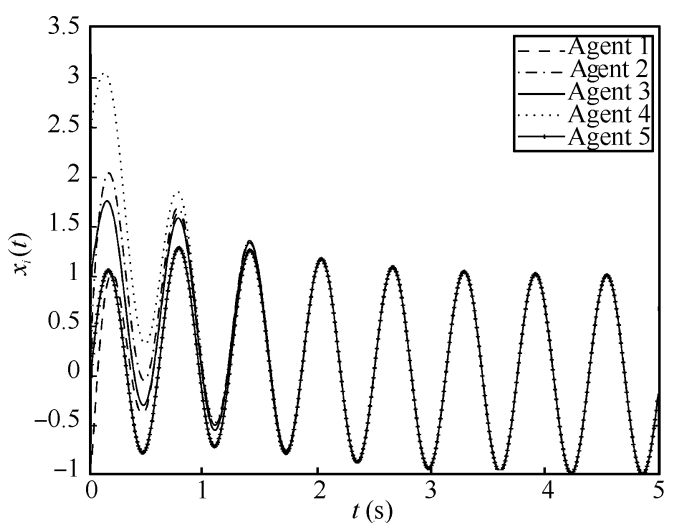

(b) State trajectories with $x(0)=[-1,0.2,1,2.5,0]^{\mathrm{T}}$

Fig. 3 The state trajectories of five agents with $x_{0}=\sin (t)$

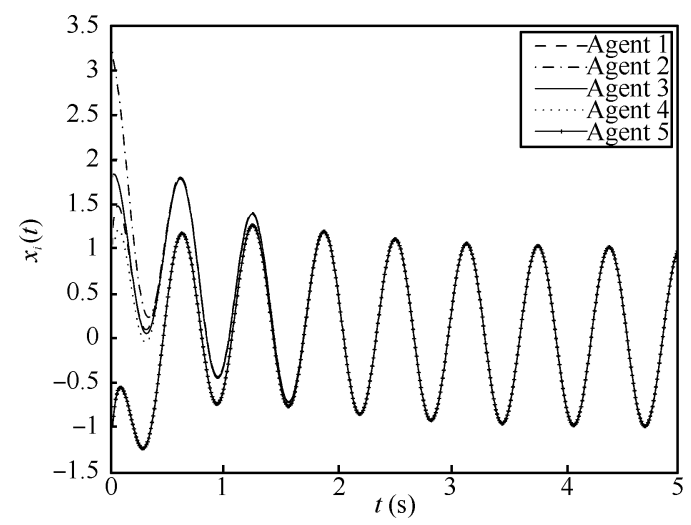

(a) State trajectories with $x(0)=[1,3.2,1.8,0.5,-1.2]^{\mathrm{T}}$ 


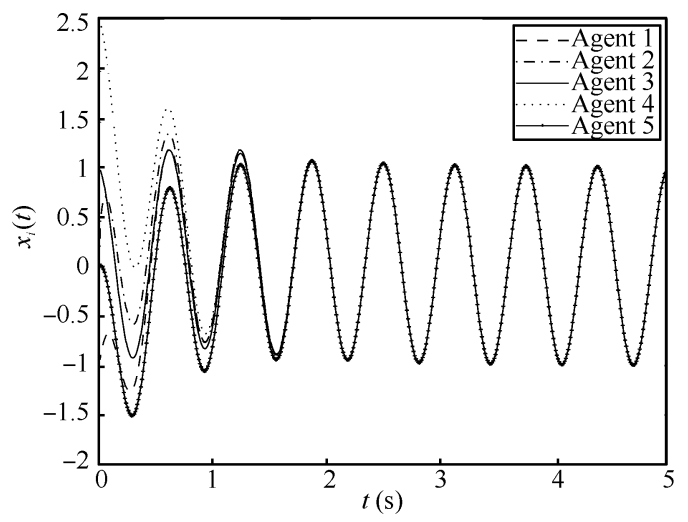

(b) State trajectories with $x(0)=[-1,0.2,1,2.5,0]^{\mathrm{T}}$

Fig. 4 The state trajectories of five agents with $x_{0}=\cos (t)$

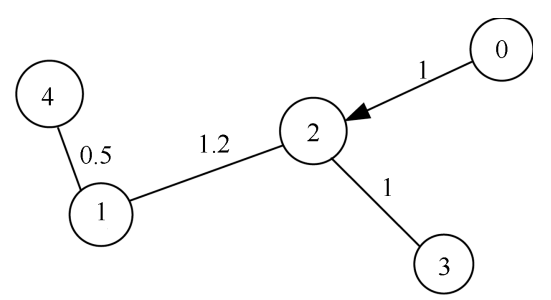

Fig. 5 The communication topology

If the second-order leader agent's states are $x_{0}=1$ and $v_{0}=0$, and the initial conditions are selected as $x(0)=[0,1,3,1.5]^{\mathrm{T}}, v(0)=[-1,0,1,2]^{\mathrm{T}}$, then the simulation results of the double integrator dynamical multi-agent systems (7) with nonlinear protocol (8) can be shown as in Fig. 6. And Fig. 7 shows that the finite-time consensus can also be achieved if the initial conditions are changed to be $x(0)=[-1,0.2,2.5,1]^{\mathrm{T}}$ and $v(0)=[1.2,-0.5,-1.5,0.2]^{\mathrm{T}}$.

Figs. 8 and 9 show the simulation results of double integrator dynamical multi-agent systems (7) with nonlinear consensus protocol (8) in different initial states, if we set the second-order leader agent's states to be $v_{0}=0.1 \cos (0.1 t)$ and $x_{0}=\sin (0.1 t)$.

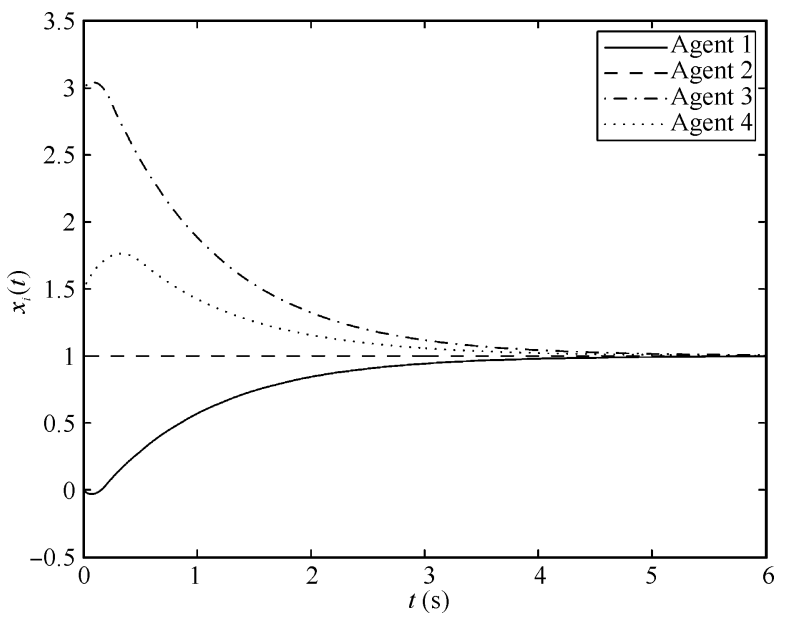

(a) Agent positions with $x(0)=[0,1,3,1.5]^{\mathrm{T}}$

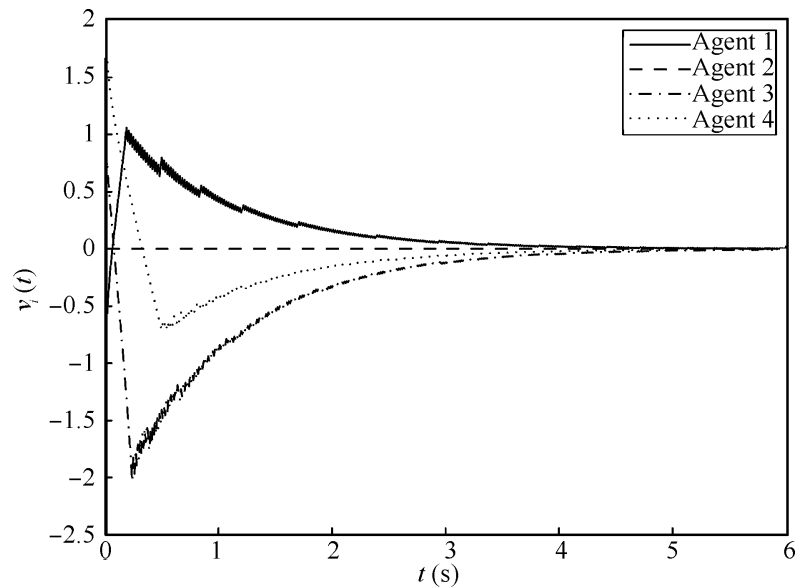

(b) Agent velocities with $v(0)=[-1,0,1,2]^{\mathrm{T}}$

Fig. 6 The trajectories of four agents with $x_{0}=1, v_{0}=0$

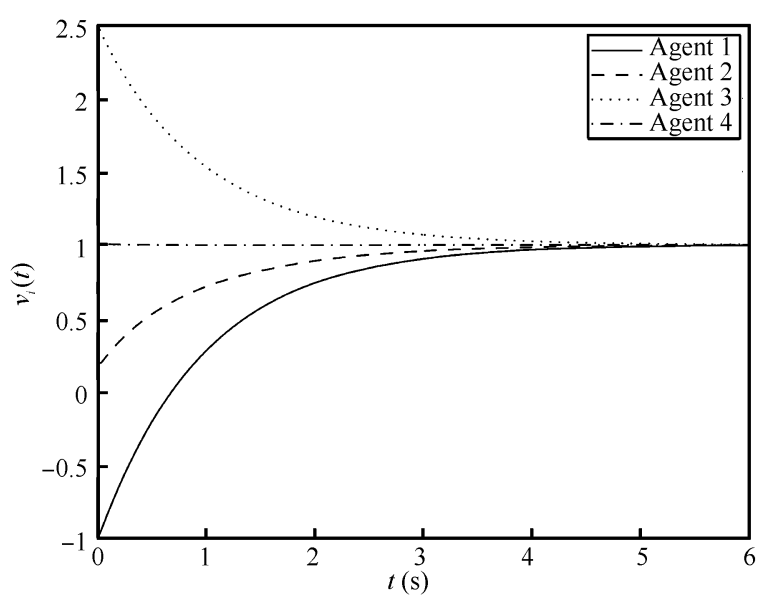

(a) Agent positions with $x(0)=[-1,0.2,2.5,1]^{\mathrm{T}}$

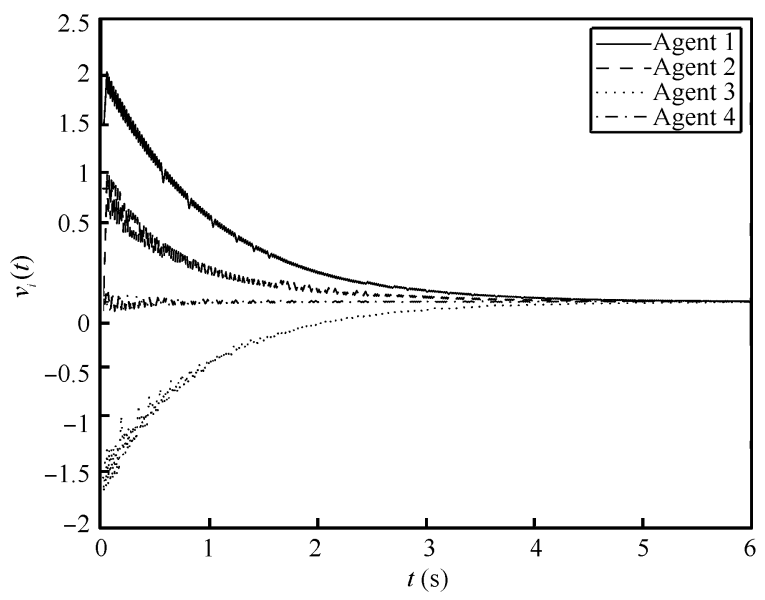

(b) Agent velocities with $v(0)=[1.2,-0.5,-1.5,0.2]^{\mathrm{T}}$

Fig. 7 The trajectories of four agents with $x_{0}=1, v_{0}=0$

Figs. 6-9 show that the consensus can be achieved in finite time under different initial states, which means a better robustness compared with other literature whose consensus states closely relate to the agents' initial states. These figures also indicate that our results are effective and have much faster convergence speed than the asymptotic results in $[6,7]$, etc. 


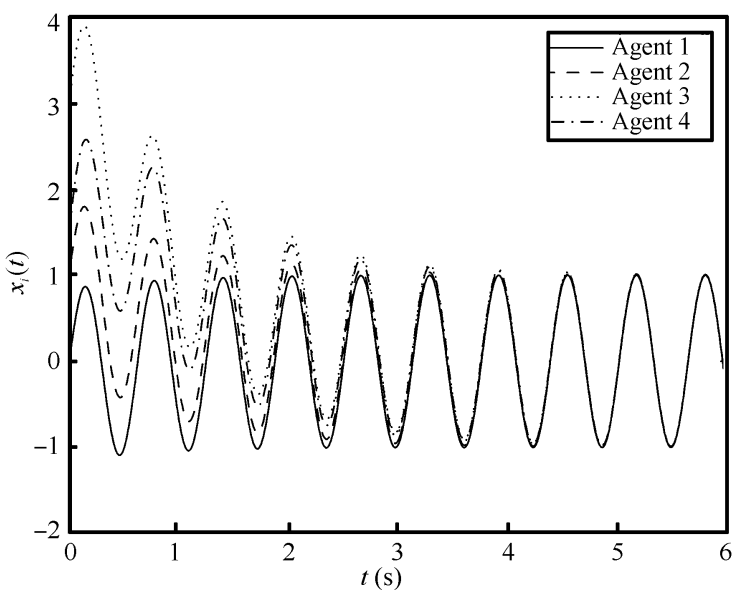

(a) Agent positions with $x(0)=[0,1,3,1.5]^{\mathrm{T}}$

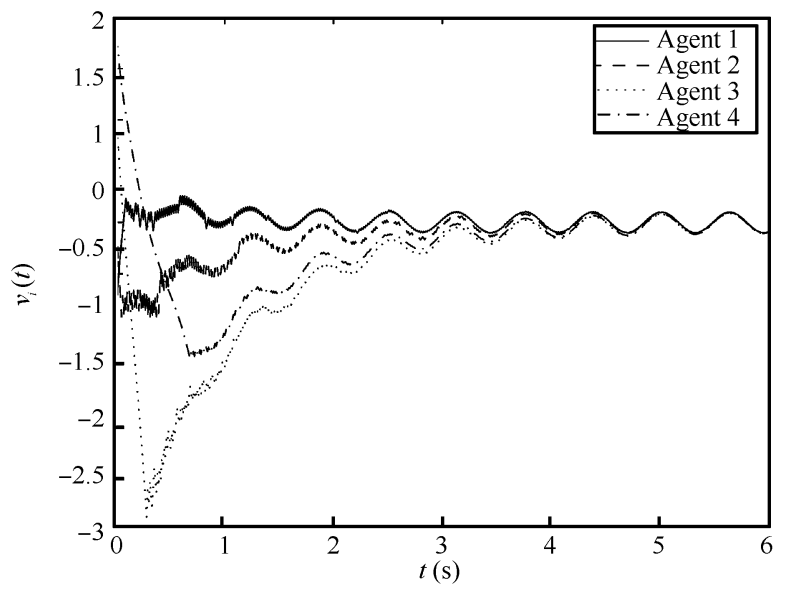

(b) Agent velocities with $v(0)=[-1,0,1,2]^{\mathrm{T}}$

Fig. 8 The trajectories of four agents with $x_{0}=\sin (0.1 t), v_{0}=$ $0.1 \cos (0.1 t)$

Figs. 2, 6, 7 indicate that the stationary consensus of multi-agent systems can be achieved in finite time via nonlinear consensus protocols. And Figs. 3, 4, 8, 9 show that the consensus can still be achieved in finite time with an active leader via nonlinear consensus protocols.

The simulation results in Sections 4.1 and 4.2 indicate the validity of the proposed nonlinear protocols, which have a faster convergence speed than the linear protocol results ${ }^{[16]}$ and asymptotic results ${ }^{[6,7]}$.

\section{Conclusions}

In this paper, we investigate the finite-time consensus problems of single and double integrator dynamical multiagent systems via nonlinear control protocols, respectively. Novel nonlinear protocols and corresponding theoretical results are proposed. Compared with the existing results, the consensus state of all agents can be achieved in finite time and remains the same if we change the initial states, which means a better robustness. Some simulation results are presented to illustrate the effectiveness of our theoretical results.

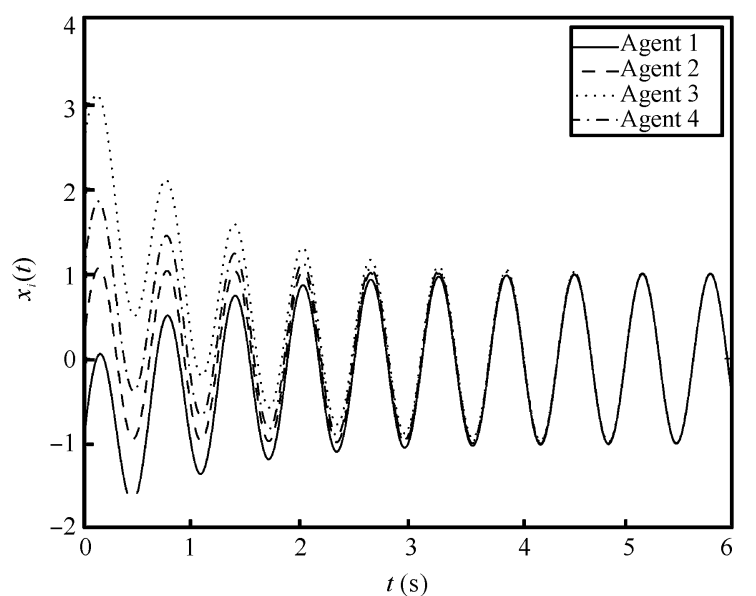

(a) Agent positions with $x(0)=[-1,0.2,2.5,1]^{\mathrm{T}}$

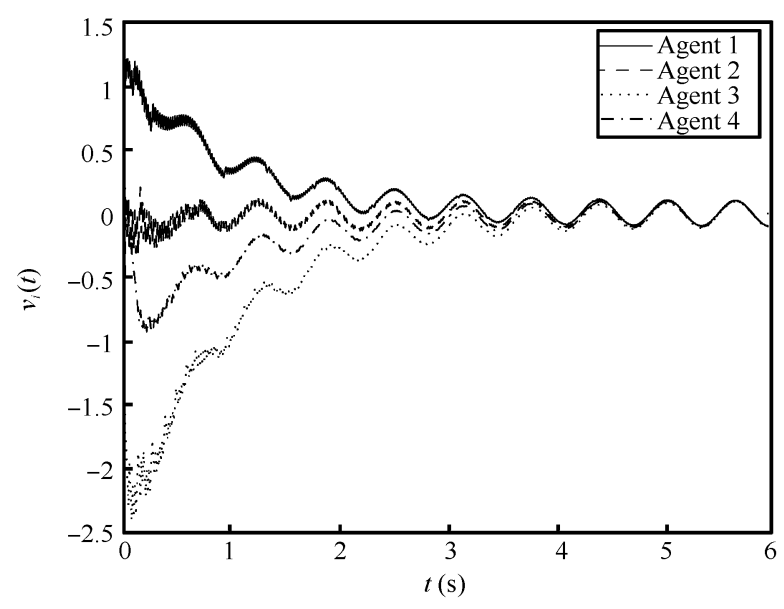

(b) Agent velocities with $v(0)=[1.2,-0.5,-1.5,0.2]^{\mathrm{T}}$

Fig. 9 The trajectories of four agents with $x_{0}=\sin (0.1 t), v_{0}=$ $0.1 \cos (0.1 t)$

\section{References}

[1] Z. Q. Wu, Y. Wang. Dynamic consensus of high-order multiagent systems and its application in the motion control of multiple mobile robots. International Journal of Automation and Computing, vol. 9, no. 1, pp. 54-62, 2012.

[2] E. F. Yang, D. B. Gu. Nonlinear formation-keeping and mooring control of multiple autonomous underwater vehicles. IEEE/ASME Transactions on Mechatronics, vol.12, no. 2, pp. 164-178, 2007.

[3] J. Yan, X. P. Guan, X. Y. Luo, F. X. Tan. Target tracking and obstacle avoidance for multi-agent networks with input constraints. International Journal of Automation and Computing, vol. 8, no. 1, pp. 46-53, 2011.

[4] W. Ren, R. W. Beard. Consensus seeking in multiagent systems under dynamically changing interaction topologies. IEEE Transactions on Automatic Control, vol.50, no. 5, pp. 655-661, 2005.

[5] M. H. Zhu, S. Martínez. Discrete-time dynamic average consensus. Automatica, vol. 46, no. 2, pp. 322-329, 2010. 
[6] D. Bauso, L. Giarré, R. Pesenti. Non-linear protocols for optimal distributed consensus in networks of dynamic agents. Systems \& Control Letters, vol.55, no.11, pp.918-928, 2006.

[7] J. Cortés. Distributed algorithms for reaching consensus on general functions. Automatica, vol.44, no. 3, pp.726-737, 2008 .

[8] P. Lin, K. Y. Qin, H. M. Zhao, M. Sun. A new approach to average consensus problems with multiple time-delays and jointly-connected topologies. Journal of the Franklin Institute, vol. 349, no. 1, pp. 293-304, 2012.

[9] G. Q. Hu. Robust consensus tracking of a class of secondorder multi-agent dynamic systems. Systems \& Control Letters, vol. 61, no. 1, pp. 134-142, 2012.

[10] X. W. Liu, T. P. Chen, W. L. Lu. Consensus problem in directed networks of multi-agents via nonlinear protocols. Physics Letters A, vol. 373, no. 35, pp. 3122-3127, 2009.

[11] L. Wang, F. Xiao. Finite-time consensus problems for networks of dynamic agents. IEEE Transactions on Automatic Control, vol. 55, no. 4, pp.950-955, 2010.

[12] F. C. Jiang, L. Wang. Finite-time weighted average consensus with respect to a monotonic function and its application. Systems \& Control Letters, vol. 60, no. 9, pp. 718-725, 2011.

[13] S. H. Li, H. B. Du, X. Z. Lin. Finite-time consensus algorithm for multi-agent systems with double-integrator dynamics. Automatica, vol. 47, no. 8, pp. 1706-1712, 2011.

[14] F. C. Jiang, L. Wang. Finite-time information consensus for multi-agent systems with fixed and switching topologies. Physica D: Nonlinear Phenomena, vol.238, no. 16, pp. 1550-1560, 2009.

[15] G. Hardy, J. Littlewood, G. Polya. Inequalities, Cambridge, UK: Cambridge University Press, 1952.

[16] R. Olfati-Saber, R. M. Murray. Consensus problems in networks of agents with switching topology and time-delays.
IEEE Transactions on Automatic Control, vol.49, no. 9, pp. 1520-1533, 2004.

[17] S. P. Bhat, D. S. Bernstein. Finite-time stability of continuous autonomous systems. SIAM Journal on Control and Optimization, vol. 38, no. 3, pp. 751-766, 2000.

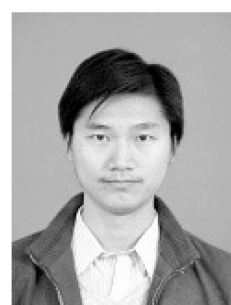

networks.
Ya-Kun Zhu received his bachelor degree in applied mathematics from Henan Normal University, China in 2006, and his master degree from Wenzhou University, China in 2009. Now, he is a Ph. D. candidate in control theory and control engineering at Yanshan University, China.

His research interests include cooperative control of multi-agent systems and wireless

E-mail: ykzhu@ysu.edu.cn (Corresponding author)

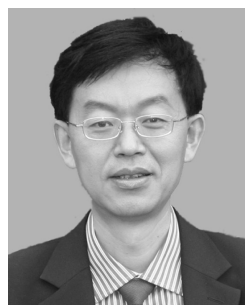

Xin-Ping Guan received his master degree in applied mathematics in 1991, and Ph. D. degree in electrical engineering in 1999, both from Harbin Institute of Technology, China. Since 1986, he has been at Yanshan University, where he is currently a professor of control theory and control engineering. In 2007, he also joined Shanghai Jiao Tong University, China.

His research interests include robust congestion control in communication networks, cooperative control of multi-agent systems, and networked control systems.

E-mail: xpguan@ysu.edu.cn

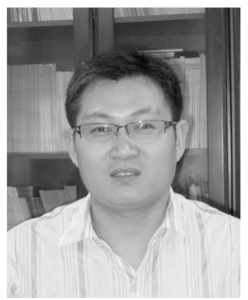

Xiao-Yuan Luo received his $\mathrm{Ph}$. D. degree from the Department of Electrical Engineering, Yanshan University, China in 2005. Now, he is a professor at Yanshan University, China.

His research interests include multiagent systems and networked control systems.

E-mail:xyluo@ysu.edu.cn 